\title{
Route Compaction for Directional Route Discovery in MANETs
}

\author{
Vinay Kolar ${ }^{\dagger}$, Paul Rogers ${ }^{\ddagger}$ and Nael B. Abu-Ghazaleh ${ }^{\dagger}$ \\ CS. Department, Binghamton University \\ Binghamton, NY 13902-6000 \\ $\dagger$ \{vinkolar, nael\}@C. . binghamton .edu \\ ${ }^{\ddagger}$ progers1@binghamton. edu
}

\begin{abstract}
Route discovery in reactive routing protocols for MANETs use flooding to disseminate route requests. Such operations rely on MAC level broadcasts to reach all nearby nodes without prior knowledge of their identity. For networks with directional antennas, this creates the following challenge: only neighbors in omni-directional range are discovered, leading to long paths and suboptimal operation. To address this problem sweeping directional MAC broadcasts are often used. Sweeping broadcasts have a high overhead; in addition, we observe that they can cause suboptimal route discovery, especially under high loads. To address these shortcomings, we propose a new directional route discovery approach called Route Compaction. Route compaction relies on enhanced version of omni-directional route discovery to find paths, avoiding the problems with sweeping broadcast. Route compaction then attempts to compact routes by replacing multiple hops with a single directional hop whenever possible. Our experiments show that this approach provides excellent directional route discovery capability at a lower overhead than sweeping broadcast.
\end{abstract}

Index Terms - Ad hoc networks, Directional Antenna, Routing.

\section{INTRODUCTION}

Ad hoc networks typically use Omni-directional Antennas (OAs) because they are commodity and inexpensive. Directional Antennas (DAs) provide superior performance to OAs. For a given energy cost, the range of a directional transmission is significantly longer than that of an omni-directional transmission. As a result, the number of hops needed to reach a given destination is lower; a shorter path translates to higher capacity, lower energy consumption per delivered packet, and shorter end to end delay [1]. Moreover, the focused beam helps to reduce the interference caused by the signal in other directions increasing channel reuse.

There are several unique characteristics of DAs that pose challenges to protocol designers. One of the problems occurs because MANET MAC level broadcasts, an operation heavily used in routing protocols, is carried out using omni-directional transmission. In particular, route discovery algorithms ${ }^{1}$ typically employ MAC broadcasts. A Route REQuest (RREQ) packet is broadcast throughout the network until it reaches the destination which then sends Route REPlies (RREPs) setting up the routing information. Using such an approach

\footnotetext{
${ }^{1}$ Although we use flooding in this paper, we believe the proposed solutions can be customized for other Network Wide Broadcast algorithms [2], [3].
}

in a DA network leads to the following inefficiency: omnidirectional broadcasts will only discover neighbors that are in omni-directional range - routes that use directional neighbors (those reachable only through a directional transmission) will not be discovered. This leads to the use of suboptimal routes and lower overall network performance [4]. More detailed background information is provided in Section II.

Choudhury et al. [4] characterize the effect of using suboptimal routes due to the omni-directional discovery problem and propose a solution called Sweeping Broadcast (SB). SB requires "sweeping" the beam sequentially across all sectors transmitting directionally in each sector. The idea is similar to the "Circular RTS" proposed by Korakis in [5]. Since the broadcast is carried out at directional power, directional neighbors can be discovered. However, we show that this approach has high overhead and may not discover routes efficiently in the presence of interference. Other researchers have examined the interaction between routing and DAs. Nasipuri et al. [6] propose an optimization for directional route discovery when the general vicinity of the destination is known. Spyropoulos et al. [7] uses the shortest path based link metrics to find the optimal route in which the packets need to be sent. Saha et al. [8] discusses the use of higher range of directional antenna to overcome route breaks caused by network partition and path breaks. The above mentioned studies does not solve the problem of route discovery. We motivate the problem and discuss SB in more detail in Section III.

The primary contribution of this paper is a new approach for discovering efficient directional routes using omni-directional broadcasts and route compaction. More specifically, our algorithm first performs route discovery in a way similar to omnidirectional antennas (but enhanced to discover more routes). We follow that with a step where routes are compacted by using directional hops to replace multiple omni-directional ones. Another contribution of the paper is an omni-directional route discovery algorithm for discovering multiple routes that is significantly more efficient than existing multi-path discovery solutions. We show that our combined solution provides path quality on par or better than Sweeping Broadcast, at a significantly lower overhead. Moreover, the quality of the discovered paths is significantly better than SB in the presence of interference. The proposed solutions and the associated design space are described in detail in Section IV. We outline and experimentally evaluate the design space in Section V. 
Finally, Section VI presents some concluding remarks.

\section{BACKGROUND}

There are two types of Directional Antennas (DAs): (1) Switched Beam; and (2) Steerable. In switched beam antennas, space is divided into a fixed number of equal-size sectors. If the node knows the sector in which the next hop node is situated, then it will transmit the signal in that particular sector. In contrast, steerable antennas are able to focus their transmission power in any direction continuously (i.e., not only in the preset directions as with switched-beam). We assume a switched beam antenna model, but our solutions can be adapted to a steerable antenna environment.

To enable the use of DAs at the physical layer, a directional MAC protocol which exploits their features is needed. We use a directional MAC protocol called DMAC which uses an RTSCTS handshake similar to the IEEE 802.11 protocol [9]. More specifically, DMAC transmits directionally if the direction of the destination is known; otherwise, it transmits omnidirectionally. Furthermore, broadcast packets are transmitted omni-directionally. DMAC tracks the direction in which a node's neighbors are located using an "Angle of Arrival" Cache (AoA cache). This cache is populated and updated with every transmission heard by a node. When sending a packet, the AoA cache is queried for the angle corresponding to the next hop destination node. If a cache miss occurs, the packet is transmitted omni-directionally. When a directional transmission is not successful, is it is retried up to a retransmission limit; if the limit is reached, the cached entry is purged and packet transmission is attempted omni-directionally.

DA operation has some unique properties that have important implications on protocol performance [10]. Deafness, is the problem that occurs because a node that is transmitting or receiving directionally, is deaf towards other directions. This may cause the node not to receive transmissions sent to it (both unicast and broadcast packets). Other problems include a different type of hidden and exposed terminal problems that arise because neighbor interaction is not only a function of the distance between nodes but also to their relative locations. A Head of Line Blocking problem occurs due to the fact that a single outgoing FIFO queue is used. If the packet at the top of the queue cannot be transmitted because its sector is busy, transmission halts even though there may be packets that are destined to a different (and free) direction [11]. Finally, the problem we target in this paper is directional route discovery which occurs due to the mismatch between omni-directional and directional transmission ranges.

\section{Problem Definition and Motivation}

In this paper, we consider route discovery in Reactive Protocols for MANETs with directional antennas. In reactive protocols, a source initiates a route request flood when it needs a path to a destination (e.g., [12]). The alternative approach, proactive protocols, attempts to maintain up-to-date routing information among all nodes in the network proactively (e.g., [13]). The overhead of reactive protocols is significantly lower than proactive algorithms where many of the updates are for unused paths [14].

Route request floods are implemented using MAC level broadcast operations. However, omni-directional transmission does not have the same range as directional transmission. Accordingly, standard route request floods fail to discover paths that use hops reachable by directional transmission but not omni-directional one. The average length of discovered paths can be significantly higher than optimal. For example, this behavior would result if we take a protocol like DSR and deploy it in a directional antenna environment.

An existing solution that addresses this problem is the use of Sweeping Broadcast (SBs) where an omni-directional MAC broadcast transmission is replaced by $S$ directional broadcasts, one for each sector. Using this approach as the basis of NWB allows directional routes to be discovered. While SB should be able to discover good quality routes, it has a high overhead. The Selective Forwarding optimization, which forwards the route request flood in sectors away from the receiving sector, reduces this overhead, but may miss some routes. Moreover, since multiple sequential transmissions are needed by each node, this may result in a delay in discovering routes. We also observed in our experiments several situations where sweeping broadcast does not discover optimal routes, especially under high load. One problem is the order of the broadcast sweep. Since the packet is swept in a serial fashion, it will be received by neighbors in an order that matches their relative direction. This biases the routes found in favor of these neighbors if they reach an intermediate neighbor that is needed for an optimal route before other nodes can, the search will fail to find the optimal route (this is because the flood packet is only forwarded the first time it is received).

A similar problem occurs due to the antenna side lobes ${ }^{2}$ A transmission can be received by nearby neighbors on the side lobes. Since the side lobe power cannot reach directional neighbors, this gives nearby neighbors an advantage in the route discovery. Routes that use these nearby neighbors are suboptimal (they do not use the longer directional range and will generally require more hops). Furthermore, they may prevent optimal routes from being discovered if their rebroadcast reaches other nodes first.

Sometimes, optimal routes are not discovered because of interference (manifested as deafness and collision losses as well as sector skipping). Sector skipping is an effect which occurs during the sweeping broadcast when a particular direction is sensed to be busy. The direction is retried at the end of the sweep, and if it is still busy, the sector is skipped. In any of these cases, potential links are not discovered, leading to the possibility of suboptimal routes.

\section{SOlution Overview - Route COMPaction}

We propose an alternative approach for directional route discovery called Route Compaction. The approach has two phases: (1) Omni-directional route discovery; and (2) Route

\footnotetext{
${ }^{2}$ A directional antenna does not cover only an ideal sector. In reality, it has a primary lobe that covers the sector, and side lobes.
} 


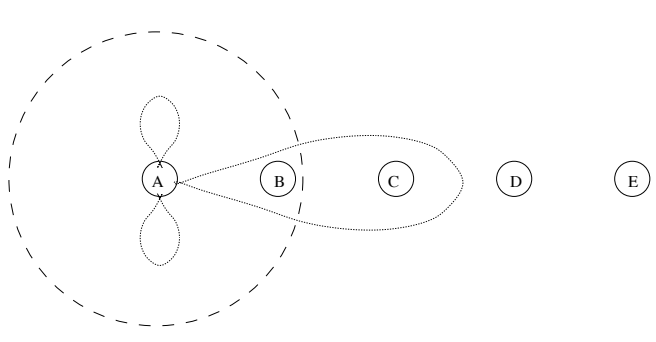

Fig. 1. Passive compaction

compaction. In the base scheme, route discovery is accomplished via standard flooding. In the route compaction phase, we shorten the received routes by attempting to eliminate intermediate hops that are not needed; more specifically, if A-B-C is a sequence in the path, and A can reach $\mathrm{C}$ via directional transmission, the path is shortened by eliminating B.

The motivation for using omnidirectional floods is to overcome the disadvantages of SBs outlined in the previous section: (1) the overhead is reduced; (2) the problems due to the sequential order of the sweeps, and the side lobes are eliminated; (3) deafness and sector skipping problems are eliminated and collision effects are reduced; and (4) delay is shortened. Meanwhile, route compaction overcomes the major disadvantage of omnidirectional route discovery - inflated routes because of inability to discover directional neighbors.

A limitation of route compaction is that it requires omnidirectional connectivity - in networks that are sparse with nodes that are only reachable directionally, omni-directional floods will fail to discover routes. However, in such situations, it is possible to switch to sweeping broadcast as a safety net either locally or at the source. We do not pursue such hybrid approaches further in this paper.

The proposed approach faces several challenges both in the route discovery and route compaction phases. Omnidirectional route discovery must discover paths suitable for compaction: an omni-directional sequence A-B-C may not be compactable into $\mathrm{A}-\mathrm{C}$ when $\mathrm{A}$ and $\mathrm{C}$ are not in directional range or when they do not know of each other's existence. This problem is worsened by the fact that flooding provides only partial discovery of the topology: many links and paths are not discovered because only the first flood packet received by a node is rebroadcast [15]; this effect may prevent discovery of compactable paths. The remainder of this section discusses these issues in more depth in the context of DSR [12]. However, route compaction applies to other reactive routing protocols. For example, distance vector compaction can be accomplished by including the cost to reach the destination in the packet header (allowing other nodes to compact their routes if your advertised route is cheaper).

\section{A. Route Compaction}

Given a route represented as an ordered sequence of the nodes that a packet goes through to get from a source to a destination, route compaction attempts to replace multiple omni-directional hops with one directional hop. Consider the nodes as shown in Figure 1. Let there be a connection between nodes $\mathrm{A}$ and $\mathrm{E}$. Let us assume that node placement is such that each adjacent node is reachable by omnitransmission, but the second adjacent node is reachable only by directional transmission. The optimal route for packet transmission will be a 2-hop path $[A-C-E]$. Because of omni-directional route discovery, the path found by node A will be $[A-B-C-D-E]$. With compaction, source node $A$ transmits directly to $\mathrm{C}$ eliminating a hop. Intermediate nodes can take the same action, thus transmitting the packet over shorter routes. We focus on two aspects of the design space in route compaction: (1) Compaction implementation alternatives; and (2) Directional Neighbor Discovery.

1) Compaction Implementation Alternatives: Compaction may be carried out by intermediate nodes: intermediate routers attempt to to take a "leap instead of a hop". When a packet arrives at an intermediate hop, it tries to find the a node in the source route which is nearest to the destination and which can be reached by just one hop. If such a node is found, then the packet is directly sent to it rather than sending it to the next hop as specified by the source route. Such compaction is carried out transparently to the source. Note that compaction will shorten an existing path but not discover a shorter path that is not a subset of the active path.

Alternatively, compaction may be carried out by informing the source of the new route using Gratuitous Route Replies (Gra-RREP); Gra-RREPs are part of the DSR draft [12]. GraRREPs are generated when the node realizes that it has a shorter path than the one in the source route included in the packet. An issue with this alternative is the overhead that occurs due to gratuitous replies. This approach is susceptible to redundant Gra-RREPs sent back to the source by different intermediate nodes which have the same path in their cache. Standard techniques are used to reduce this effect (e.g., restricting Gra-RREPs to nodes belonging to the replied path, using random backoff and canceling the scheduled send if other Gra-RREPs are overhead, or scheduling the GraRREPs with a small probability). We use the gratuitous reply implementation for most of the proposed protocols.

2) Directional Neighbor Discovery Alternatives: The key capability needed for compaction is the knowledge of directional neighbors. The DMAC layer has partial knowledge of directional neighbors that is collected in the AoA cache as directional transmissions are overheard. We keep the directional neighbor tracking responsibility to the MAC layer and propagate up to the routing layer via upcalls. Since the neighbor information in the AoA cache is restricted to overheard neighbors, it is incomplete; more complete neighbor knowledge is required for effective compaction.

Neighbor Discovery (ND) may already be carried out for other services required by the node since it is needed for functions such as topology control [16], clustering [17], and Connected Dominating Set based forwarding [3]. In addition, ND is used to track connectivity information by some routing protocols (e.g., Hello Messages in AODV [18]). Thus, requiring ND may not result in additional overhead. For cases where ND is not already present, we identify the following route compaction protocols based on the ND approach:

Passive Discovery and Compaction (PaDC): only the neighbor 


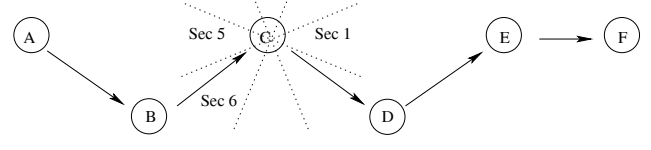

Fig. 2. Active Sector Optimization

information found in the AoA cache is used for compaction; no explicit route discovery is performed. This approach has the lowest overhead but will miss compaction opportunities.

Proactive Discovery and Compaction (ProDC): a proactive neighbor discovery is carried on in the background to populate AoA caches. This approach has a high overhead comparable with sweeping broadcast - but will result in effective compaction. In the base implementation, ND is initiated periodically at all nodes; the period may be static or dynamically adjustable to reflect mobility and other changes. When ND is triggered a node sweeps all sectors with a short transmission. Receiving nodes now learn about the sending node; this information is up-called to the routing layer to allow compaction. In our implementation, the discovery period must be related to the AoA cache TTL time to prevent entries from expiring because ND is being carried out at too low a frequency. Clearly, this approach has significant overhead; later we propose a more efficient active sector optimization.

Reactive Discovery Compaction (ReDC): In the reactive approach ND is only carried out by the nodes that form the route and only for active sectors and sectors adjacent to them. This approach provides a lower overhead than proactive discovery. More importantly, it provides finer control on what nodes carry out compaction; we use this ability to compact multiple candidate routes. Furthermore, the overhead of ND can be reduced by making sure that nodes that appear in multiple paths being compacted do not attempt multiple ND; this is similar to the No-Repeat optimization of proactive ND which is described below.

Active Sector Optimization for ProDC: Consider C in Figure 2 which carries traffic in sectors towards B and D. $A$ would not passively learn of $C$. Explicit ND allows A to learn about $\mathrm{C}$ allowing it to compact the path to $\mathrm{F}$. Assuming an 8 sectored antenna, $\mathrm{C}$ has to send 8 packets at every period.

We use an optimization, called Active Sector Neighbor Discovery (ASD), to significantly reduce in the overhead of ND. In this approach, when $\mathrm{C}$ recognizes that most of the incoming traffic is in sector 6 , it will transmit the ND packets only in the sector 6 and its two adjacent sectors. This argument is geometric - paths tend to bring packets physically closer to the destination and candidate nodes for compaction will likely be clustered around the current transmission direction. Activity in a sector is measured every period relative to a configurable threshold - if this threshold is exceeded, the sector is marked for active sector discovery. As a further improvement, if $\mathrm{C}$ had sent packets along any of the marked active sectors in the past interval, it does not generate an additional transmission in that sector.

\section{B. Route Discovery for Compaction and Multi-route schemes}

Compacted path as described thus-far are restricted to be a subset of the original path being compacted. Consider the nodes as shown in the Figure 3 where each node is separated by a fixed distance from its horizontal and vertical neighbor; adjacent neighbors as well as diagonal neighbors are reachable by omni-transmission. Further, assume that node 1 can reach node 3 directly via directional transmission, but not omni-directionally. The routes from 7 to 12 include $[7-8-9-10-11-12], \quad[7-14-21-16-11-12]$ and $[7-2-9-16-17-12]$. These routes are equal from an omni-directional perspective since they all have the same hop count. The optimal route has a length of 3 hops but its only achievable when the straight line route is compacted. If the source transmits the packet along the routes that alternate sectors, it is not possible to reach the optimal path.

It is not always true that a "straight" route provides the best compaction. Moreover, the best directional route may result from a compacted route that is not optimal before compaction. Thus, a critical component of this approach is the ability of the source to discover and compact appropriate routes: routes that will be near optimal once they are compacted. In general, it is not possible to predict such routes without knowledge of node locations and reachability. Compacting multiple routes results in a higher chance of discovery of optimal directional routes. The route discovery process should uncover sufficient candidate routes. Since omni-directional flooding is unable to sufficiently discover the route topology [15], a more effective route discovery approach is needed. This is the second component of the proposed approach.

Effective Route Discovery: The target of this component of the solution is uncover sufficient routes to allow effective compaction at a low overhead. The poor coverage of flooding occurs because the flood packet is rebroadcast only the first time it is received: effectively, only one incoming link per node is discovered. This problem is known and solutions for it exist; unfortunately, existing solutions are expensive [15]. We propose a more efficient alternative called Multi-Route Attachment(MRA).

Multi-Route Attachment(MRA): Existing solutions for multiple path discovery attempt to find all routes. In the basic solution, each node propagates the RREQ flood every time it receives a packet with a new path in it. This enables the destination to receive multiple RREQs with different routes, providing full discovery of the links along the way to the destination. Unfortunately, the overhead of RREQ flood in this case grows at an exponential rate for each forwarded RREQ. Moreover, large overhead is incurred as the replies for the large number of discovered routes are sent independently. Heuristics can be used to reduce these overheads but sacrifice some coverage.

We propose a Multi-Route Attachment(MRA) scheme that provides multiple route discovery at a significantly lower overhead than existing approaches. In this scheme, the initial forwarding of the RREQ with each node recording all the RREQ packets it receives but forwarding only the first. When the destination replies with an RREP, intermediate nodes also include the other paths known by them. The RREP remains 
a single unicast packet that travels along the reverse path. Some paths will not be covered; while sacrificing some path coverage, we are able to discover many of the paths at a much lower overhead than the existing solutions. The overhead is comparable to that of traditional route discovery (same number of transmissions, with larger RREP packets), but much higher route coverage is achieved.

For example, consider the source node 7 trying to reach the destination 12 in the Figure 3. Let us assume that node 17 has heard two RREQs with paths [7-8-9-10-17] and [7-14-21-22-17]. Let the RREP travel from Node 12 contain the reply path as $[7-8-9-10-17-12]$. As the RREP reaches node 17 , it will try to combine the routes that it has learnt from RREQs and would send the RREP to its next hop node 10 with paths $[7-8-9-10-17-12]$ and [7-14-21-22-17-12]. It can be seen that routes that are spread out can be captured by such combinations.

Note that using a link route cache (a cache that tracks available links, and constructs route from them dynamically) should be able to recover many more paths than a path based cache. Furthermore, if a link cache is assumed, intermediate nodes can avoid including their information on every RREP and include it only on the first one, further reducing the overhead. We are currently pursuing a link-cache implementation of multi-route attachment.

Selection of possible routes which can be tried for compaction is currently done by selecting a random route among the unused pool of discovered routes. As a part of future work, more intelligent route selection policies like "maximal node disjointness" can be used to select a wider variety of paths.

\section{Dependence of route compaction on MRA}

The timing and the route taken by RREP contribute to the effectiveness of the MRA, thus influencing the variety of the routes available at the source. The attachment of routes in MRA relies on characteristics of the RREPs. If the RREP is generated too early, before the RREQ flooding subsides, then the intermediate nodes would have a limited knowledge about the routes to the source. This restricts the number of routes that are attached. Since it takes larger distance for the RREQs to reach nodes which are near the destination, such behavior can be observed more near the destination.

Multiple RREPs can be generated when the destination receive RREQs from multiple neighbors. The route attached in such RREQs will be reversed to send the RREP. The path taken by the RREPs also contribute to the variety of the routes that are reported by MRA. If two or more RREPs travel on the path which is has minimal common nodes, then the diversity of routes informed by MRA is higher.

\section{Performance Evaluation}

This section presents an evaluation of the proposed compaction protocols and compares them against Sweeping Broadcast. We use the QualNet [19] simulator using the Directional MAC (DMAC) protocol. Table I lists the relevant simulation parameters.

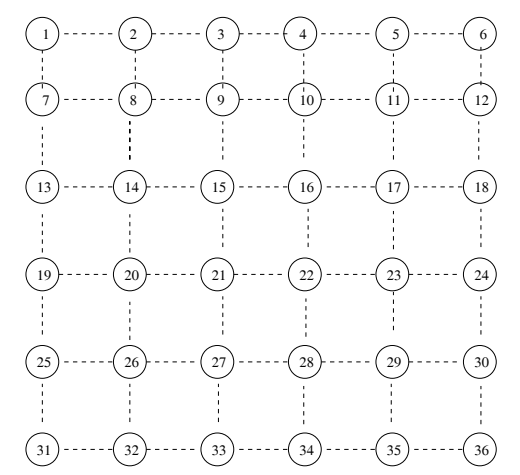

Fig. 3. Grid topology

\begin{tabular}{|l|l|}
\hline Parameter & Value \\
\hline Omni-directional range & $250 \mathrm{~m}$ \\
Directional range & $344 \mathrm{~m}$ \\
Directional antenna model & Switched beam \\
Path loss Model & Two Ray \\
Transmission power & $24.5 \mathrm{dBm}$ \\
Directional gain & $10.0 \mathrm{~dB}$ \\
\hline
\end{tabular}

TABLE I

SIMULATION PARAMETERS

\section{A. Comparison of Compaction protocols}

A grid topology as shown in the Figure 3 is used for the first set of experiments. Nodes are uniformly placed such that each node is able to receive the packet from its second adjacent node either in horizontal or vertical direction. Diagonal nodes can be reached by omni-directional transmission and directional transmission along diagonal cannot reach nodes beyond the immediate diagonal node. For example, Node 1 can reach node 8 by omni transmission but cannot reach node 9 or node 15 by directional transmission. In the given grid scenario, the optimal hop count is 5 for omni-directional transmission and 3 for directional for connections running along the grid lines. It can be observed that routes only along the grid lines can be compacted. If the route taken is non-linear, optimal compaction cannot be done. We first measure the effectiveness of compaction was measured under this strict topology because it allows direct prediction of optimal routes.

We first evaluate the proposed compaction protocols. The performance metrics we use are the path inflation (average path length relative to the best available one) and overhead. Figure 4(a) shows the path inflation relative to the optimal value of 3. The overhead of the PaDC protocol is very low as shown in the Figure 4(b) because of its passive nature. However, PaDC fails to effectively compact the routes because of its dependence on route geometry. The ProDC protocol triggers ND packets at regular intervals. Optimizations to ProDC, as shown by Opti-ProDC in Figure 4, protocols as explained in Section IV-A.2 achieves good compaction at around $68 \%$ lesser broadcast overhead than ProDC. Figure 4(b) shows that significant broadcast overhead is incurred for proactive protocols. However, if ND is combined with other services to the node as explained in Section IV-A.2, this overhead can be eliminated. It can be seen that ReDC is very near to the optimal hop count (Figure 4(a)) with significantly lesser 


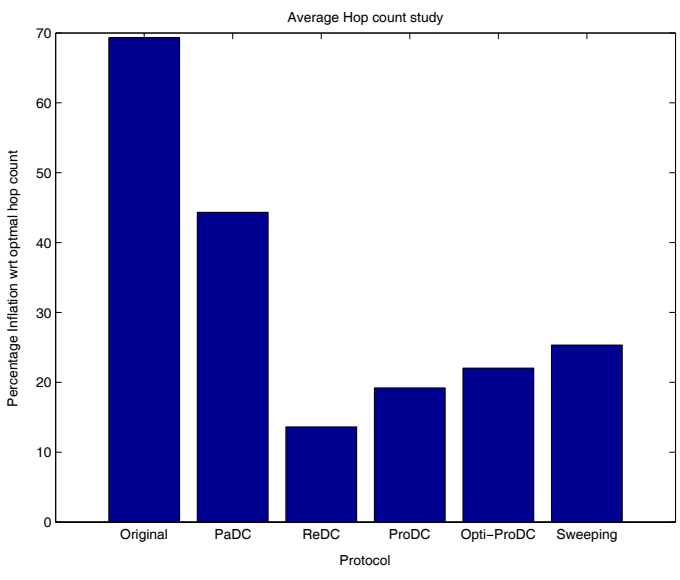

(a) Average Hop Count study

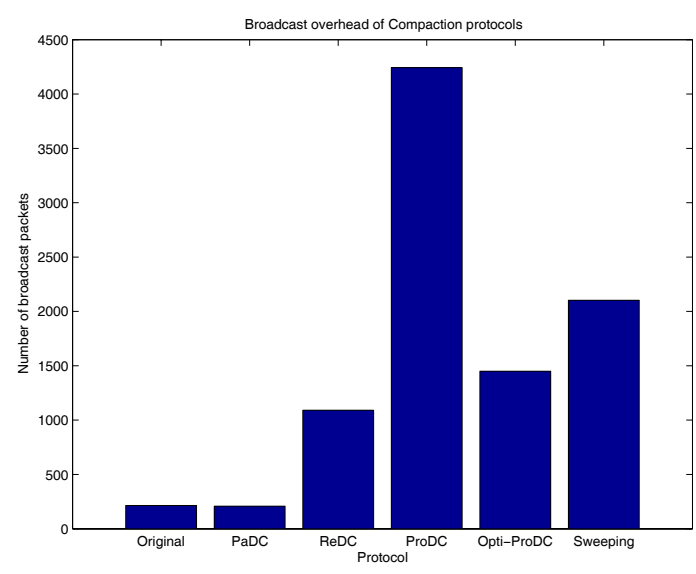

(b) Broadcast overhead study

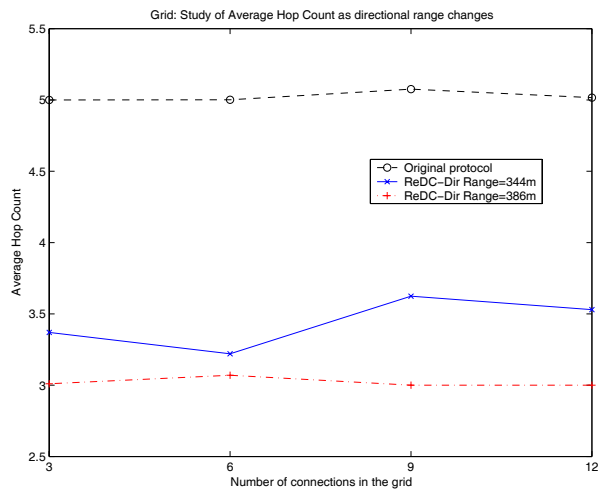

Fig. 7. Effectiveness of compaction as a function of Directional Range.

to sweeping broadcast. The broadcast overhead of ReDC is significantly better than that of sweeping as shown in Figure 6(b)(68\% better than sweeping broadcast for 100 nodes scenario). Note that as the number of sectors in an antenna is increased, the broadcast overhead of sweeping broadcast increases linearly whereas the overhead in ReDC remains stable. The end-to-end delay and transmission energy follow a similar trend to the average hop count since a lower hop count translates to lower end-to-end delay and transmission energy. The number of packet retransmits due to route failure is substantially higher in SB; we conjecture that this is due to the problems we discussed earlier (side-lobe reachability and fixed sweeping order) creating destructive interactions. The larger the number of packet retransmits, the larger are the route errors which leads to greater route maintenance overhead.

\section{Effectiveness of Compaction as a function of Directional Range:}

Compaction in the above grid scenarios were restriced along the grid lines. If the directional range is increased such that "diagonal compaction" can happen, then it is possible to compact additional routes other than the straight line routes. 6(a) shows that average hop count of ReDC is comparable
Figure 6 shows the results under random topology with 8 connections in a $1000 \mathrm{~m} \times 1000 \mathrm{~m}$ area varying the number of nodes. It can be observed in that ReDC performs considerably

\section{B. Comparison of Reactive compaction and Sweeping Broad-}

The performance of ReDC was analyzed with respect to normal DSR operation and Sweeping Broadcast (which we also implemented). Grid and Random topologies were used average end-to-end delay, goodput and broadcast overhead of various protocols under grid topology. Broadcast overhead is Note that for an antenna with $n$ sectors, $n$ packets are transmitted for sweeping broadcast. The number of connections in the grid is altered to observe the effect on different parameters. and from one end of the grid to another. It can be seen number of connections increase the ReDC performs better the Swe This is because of the sub-optimal routes found by End-to-End delay of ReDC and sweeping are comparable or lesser connections. For higher number of connections, 5(c) shows the goodput of the protocols. The goodput of sweeping broadcast droops as the number of connections significantly lesser in ReDC(around 37\% for 12 connections). The effect is more pronounced in Figure 6(b) of random scenario. Techniques similar to the optimizations for ProDC can be applied to ReDC and Selective Forwarding [4] to reduce the broadcast overhead further. 


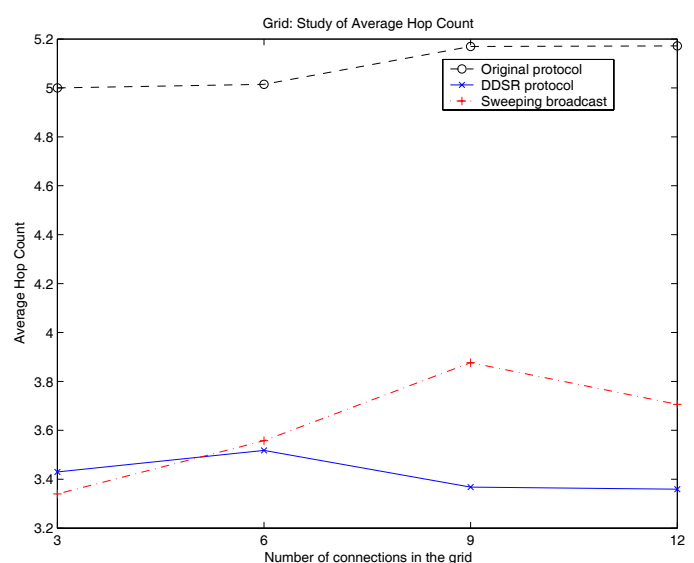

(a) Average Hop Count

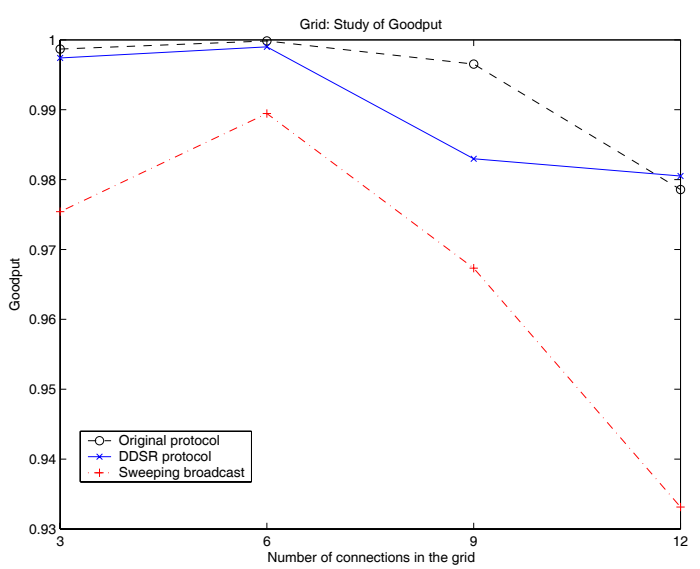

(c) Goodput study

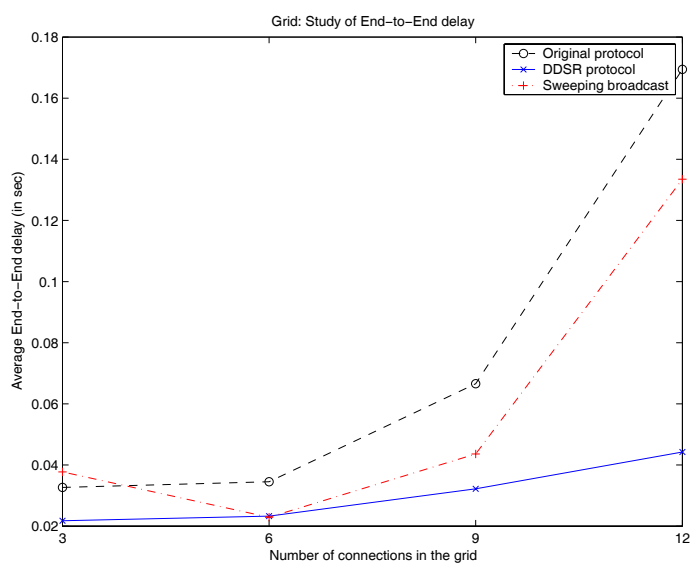

(b) End to End delay study

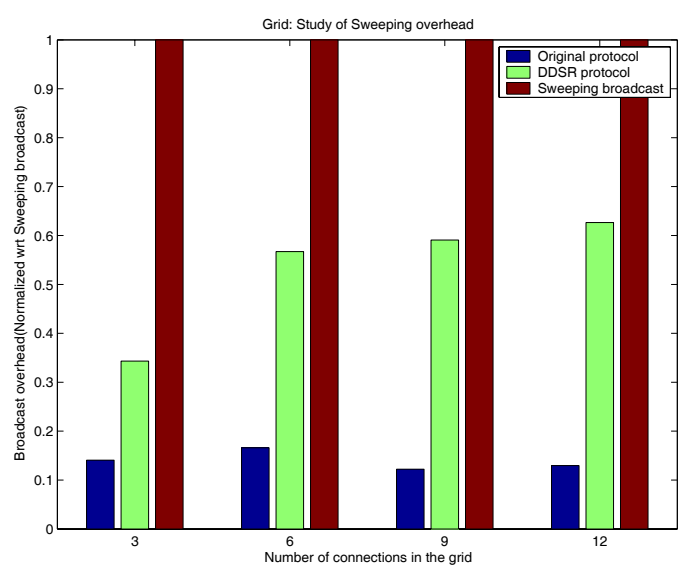

(d) Broadcast overhead study

Fig. 5. Study of ReDC protocol in Grid Topology

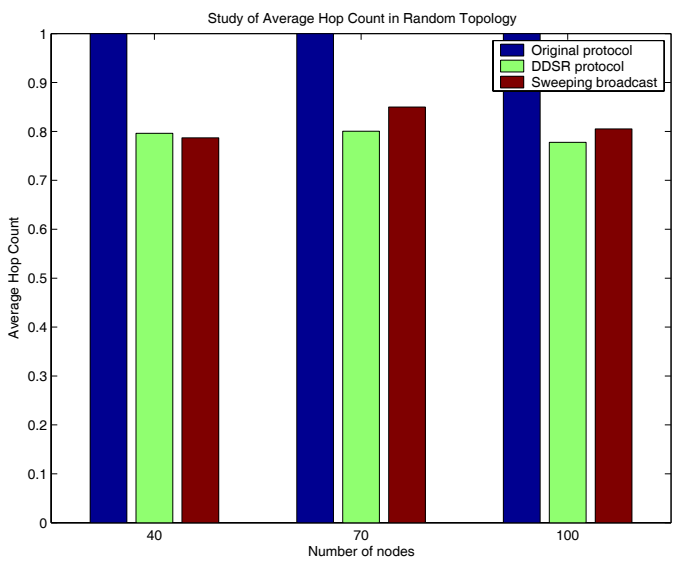

(a) Average Hop Count

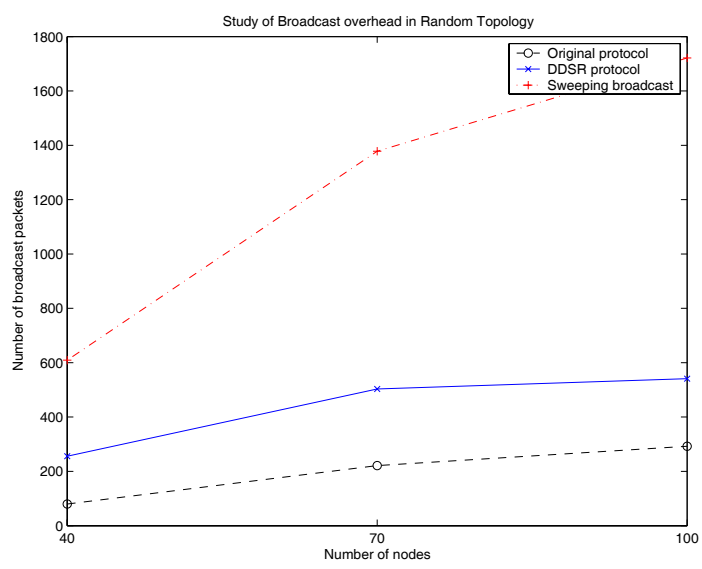

(b) Broadcast overhead study

Fig. 6. Random Topology: Study of ReDC protocols 
Figure 7 shows the effectiveness of compaction when the directional range is increased. When the directional range is stepped up to $386 \mathrm{~m}$ (node 1 can reach node 9 but not node 15 in Figure 3), the ReDC consistently performs very close to the optimal hop count of 3 . The effectiveness of the compaction increases if the number of routes that can be optimally compacted is higher.

\section{CONCLUSION AND FUTURE WORK}

This paper proposed a directional route discovery scheme to enable the utilization of the higher range of directional antennas. The existing solution to this problem, sweeping broadcast, suffers from high overhead and possible route inefficiency. The proposed approach avoids using sweeping broadcasts. It has two components: (1) we first use a modified omni-directional path discovery algorithm to allow more effective discovery of the network topology; and (2) route selection and compaction: of the discovered omni-directional paths, one or more are selected and compaction is attempted on them. Compaction attempts to replace multiple omni-directional hops with a single directional one. We studied several alternatives from the design space for both of these components. We showed that this approach provides an attractive alternative to the best known solution: it has a fraction of the overhead and is able to discover paths of equivalent quality to sweeping broadcast. Furthermore, the quality of the discovered paths is better in terms of the resulting self-interference as evidence the number of packet retransmissions along them. Although we used the DSR routing protocol, we believe that the solution can be generalized to other routing protocols.

Our future work includes the evaluation of the effect of factors such as the number of sectors and the ratio of the directional transmission power to the omni-directional one on the protocol performance. As the number of sectors increases, the overhead of sweeping would increase exponentially where as ReDC would regulate the overhead by transmitting ND packets only in selective sectors. It would be interesting to investigate the effect in future. If the neighbor is already found by other means (e.g. overhearing its transmission), the ND packets would be redundant. The ND packets transmitted are necessary for compaction but not essential whereas the sweeping packets in sweeping broadcast are essential for route discovery. Hence, we conjecture that ReDC performs much better than sweeping under high-load where the probability of a broadcast packet being lost is higher.

We are also interested in developing compaction algorithms for other routing protocols, including proactive ones. We believe the use of a link cache in place of a route cache allows significant improvement in multi-route discovery. We would also like to study the effect of mobility on compaction. Finally, we believe there is a need for an understanding of the effect of path geometry on performance. Such an understanding would assist us in Path selection heuristics that intelligently select candidate routes for compaction. It would be interesting to study the reduced broadcast overhead in compaction by integrating Neighborhood discovery schemes as suggested by Vasudevan et al. [20].

\section{REFERENCES}

[1] J. Li, C. Blake, D. S. J. D. Couto, H. I. Lee, and R. Morris, "Capacity of Ad Hoc wireless networks," in International Conference on Mobile Computing and Networking (MobiCom), 2001, pp. 61-69.

[2] Y.-C. Tseng, S.-Y. Ni, Y.-S. Chen, and J.-P. Sheu, "The broadcast storm problem in a mobile ad hoc network," Wirel. Netw., vol. 8, no. 2/3, pp. 153-167, 2002.

[3] R. Gandhi, S. Parthasarathy, and A. Mishra, "Minimizing broadcast latency and redundancy in ad hoc networks," in MobiHoc '03: Proceedings of the 4th ACM international symposium on Mobile ad hoc networking \& computing. ACM Press, 2003, pp. 222-232.

[4] R. R. Choudhury and N. H. Vaidya, "Impact of Directional Antennas on Ad Hoc Networks Routing," in Personal and Wireless Communication (PWC), 2003.

[5] T. Korakis, G. Jakllari, and L. Tassiulas, "A MAC protocol for full exploitation of directional antennas in ad-hoc wireless networks," in Proceedings of the 4th ACM international symposium on Mobile ad hoc networking \& computing. ACM Press, 2003, pp. 98-107.

[6] H. M. Asis Nasipuri, Jothsna Mandava and R. E. Hiromoto, "OnDemand Routing Using Directional Antennas in Mobile Ad Hoc Networks," in IEEE Wireless Communications and Networking Conference (WCNC), 2000.

[7] A. Spyropoulos and C. Raghavendra, "Energy efficient communications in ad hoc networks using directional antennas," in INFOCOM, 2002.

[8] A. Saha and D. Johnson, "Routing improvements using directional antennas in mobile ad hoc networks," in Rice University, CS Technical Reports, TR03-420, 2003. [Online]. Available: http://cs-tr.cs.rice.edu/ Dienst/UI/2.0/Describe/ncstrl.rice_cs/TR03-420

[9] The Editors of IEEE 802.11, IEEE Standard for Wireless LAN Medium Access Control (MAC) and Physical Layer (PHY) specifications, Nov. 1997.

[10] R. Ramanathan, "On the performance of ad hoc networks with beamforming antennas," in Proceedings of the 2nd ACM international symposium on Mobile ad hoc networking \& computing. ACM Press, 2001, pp. 95-105.

[11] V. Kolar, S. Tilak, and N. Abu-Ghazaleh, "Avoiding head of line blocking in directional antenna," in 29th Annual IEEE International Conference on Local Computer Networks (LCN'04), 2004, pp. 385-392.

[12] D. B. Johnson, D. A. Maltz, Y.-C. Hu, and J. G. Jetcheva, "The Dynamic Source Routing Protocol for Mobile Ad Hoc Networks (DSR)," February 2002. [Online]. Available: http://www.ietf.org/ internet-drafts/draft-ietf-manet-dsr-07.txt

[13] T. Clausen and P. Jacquet, "Optimized Link State Routing Protocol (OLSR)," 2003, IETF RFC 3626; http://www.olsr.net.

[14] J. Broch, D. A. Maltz, D. B. Johnson, Y.-C. Hu, and J. Jetcheva, "A performance comparison of multi-hop wireless ad hoc network routing protocols," in MobiCom '98: Proceedings of the 4th annual ACM/IEEE international conference on Mobile computing and networking. ACM Press, 1998, pp. 85-97.

[15] A. Nasipuri, R. Castaneda, and S. R. Das, "Performance of multipath routing for on-demand protocols in mobile ad hoc networks," Mob. Netw. Appl., vol. 6, no. 4, pp. 339-349, 2001.

[16] X.-Y. Li, P.-J. Wan, Y. Wang, and C.-W. Yi, "Fault tolerant deployment and topology control in wireless networks," in MobiHoc '03: Proceedings of the 4th ACM international symposium on Mobile ad hoc networking \& computing. ACM Press, 2003, pp. 117-128.

[17] S. Basagni, "Distributed clustering for ad hoc networks," in ISPAN '99: Proceedings of the 1999 International Symposium on Parallel Architectures, Algorithms and Networks (ISPAN '99). IEEE Computer Society, 1999, p. 310.

[18] C. E. Perkins, E. M. Belding-Royer, and S. R. Das, "Ad hoc On-Demand Distance Vector (AODV) Routing," January 2002. [Online]. Available: http://www.ietf.org/internet-drafts/draft-ietf-manet-aodv-10.txt

[19] "Qualnet network simulator, version 3.6," http://www.scalable-networks. $\mathrm{com} /$.

[20] S. Vasudevan, J. Kurose, and D. Towsley, "On neighbor discovery in wireless networks with directional antennas," in Proceedings of IEEE INFOCOM '05, 2005. 